\title{
Hémangiopéryctome - tumeur fibreuse solitaire maxillaire : à propos d'un cas
}

\author{
Paul Bontemps*, Muriel Brix, Etienne Simon \\ Service de Chirurgie maxillo-faciale et Chirurgie plastique, Hôpital central, Nancy, France
}

(Reçu le 18 septembre 2012 ; accepté le 22 septembre 2012)

Mots clés : tumeur fibreuse solitaire / maxillaire / immunohistochimie
Key words: solitary fibrous tumor / maxillary / immunohistochemistry
Résumé - Introduction : La tumeur fibreuse solitaire (TFS) est une tumeur rare. Elle appartient à un groupe de lésions comportant de nombreux diagnostics différentiels. Par l'étude d'un cas, les auteurs souhaitent préciser les données cliniques, paracliniques et anatomopathologiques des TFS.

Observation : Nous rapportons le cas d'une patiente, suivie depuis 1982 pour un hémangiopéricytome cérébral opéré à plusieurs reprises, adressée dans le service pour exophtalmie avec perte de l'acuité visuelle. Le CT-scan du massif facial retrouvait une récidive dans le maxillaire gauche, avec un envahissement de l'orbite homolatérale. L'artériographie préopératoire permettait d'en préciser la vascularisation et d'emboliser les branches de la carotide externe, et à minima celles de l'artère ophtalmique. La patiente a bénéficié d'une exérèse tumorale avec exentération de l'œil gauche. L'examen anatomopathologique a montré un profil morphologique et immunohistochimique (CD34, bcl-2, CD99) typique d'une TFS avec des signes d'agressivité histologique.

Discussion : Pour ce type de tumeur, c'est l'examen anatomopathologique et immunohistochimique qui apporte le diagnostic de certitude. Il existe des critères histologiques corrélés avec un potentiel de récidive locale. L'analyse microscopique permet de déterminer le potentiel agressif de la lésion. L'embolisation de la lésion a facilité l'intervention en diminuant le risque hémorragique. Des évolutions métastatiques sont rapportées dans la littérature. Les TFS nécessitent une exérèse complète pour diminuer le risque de récidive et nécessitent une surveillance prolongée à long terme.

Abstract - Hemangiopericytoma - maxillary solitary fibrous tumor: report of a case. Introduction: The solitary fibrous tumor (SFT) is a rare tumor. It belongs to a group of lesions with numerous differential diagnoses. By studying one case the authors wish to specify the paraclinical, clinical and anatomophatologic data of SFT.

Observation: We are reporting here the case of a female patient who came to us for an exophthalmos with loss of visual acuity. She had been suffering from a hemagiopericytoma since 1982 and underwent several surgeries. A CTscan of the facial massive demonstrated a relapse at the level of the left maxillary that spread to the homolateral orbit. The arteriography prior to surgery allowed to determine its vascularization and to embolism the branches of the external carotid as well as of the ophthalmic artery a minimal. The patient underwent tumoral excision with left orbital exenteration. The pathological exam showed a typical $\mathrm{H}-\mathrm{E}$ and immunoprofile of SFT, including strong CD34 reactivity. The tumor also presented microscopic criteria predictive of an aggressive course.

Discussion: In this type of tumor a confirmed diagnosis is obtained thanks to an anatomopathologic and immunohistochemical exam. There are histological criteria that correlate with a high recurrence risk. The microscopic analysis can lead to the determination of the lesion potential of aggression. The embolization of the lesion allowed an intervention with minimized hemorrhagic risks. Metastatic evolutions were observed in the literature. A complete resection is required to avoid the risk of recurrence of SFT and in addition a long term followup should be performed.

\footnotetext{
*Correspondance : paul-bontemps@orange.fr
} 


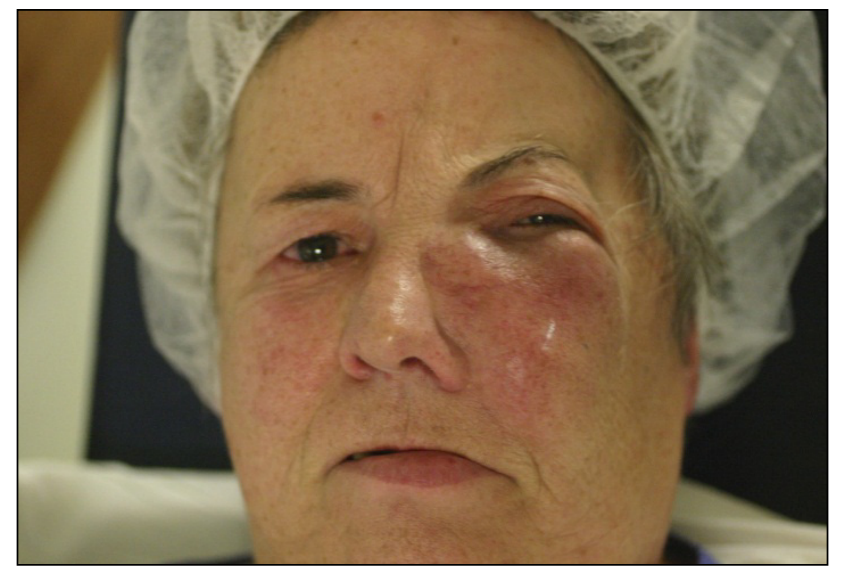

Fig. 1. Aspect pré-opératoire de la lésion.

Fig. 1. Preoperative aspect of the lesion.

Les tumeurs fibreuses solitaires (TFS) ont été décrites initialement dans la plèvre, en 1931, par Klemperer et Rabin. Cette tumeur mésenchymateuse est rare, ubiquitaire, d'évolution lente, insidieuse et dispose d'une potentialité maligne. Par l'étude d'un cas de TFS maxillaire, les auteurs souhaitent préciser les données cliniques, paracliniques et anatomopathologiques de cette lésion.

\section{Observation}

Une patiente, suivie depuis 1982 pour un hémangiopéricytome cérébral, est adressée dans le service pour une exophtalmie gauche, unilatérale, asymptomatique, d'évolution progressive. La patiente a bénéficié d'exérèses itératives. En 1982, les ophtalmologues ont réalisé l'exérèse d'une lésion se situant en arrière du globe oculaire. En 1987, 1991 et 1992 les neurochirurgiens, par voie coronale, ont effectué plusieurs exérèses. En 1992, l'exérèse chirurgicale a été complétée par une radiothérapie postopératoire. En 2010, une cinquième intervention a été réalisée pour une récidive se traduisant par une exophtalmie douloureuse associée à une extension sous-palpébrale. Un an après, la patiente s'est présentée en consultation d'ophtalmologie. L'examen clinique retrouvait une volumineuse récidive tumorale, avec envahissement du globe oculaire gauche (Fig. 1). Le CT-scan du massif facial montrait une récidive évidente de la lésion développée au dépens du plancher de l'orbite gauche entraînant une lyse osseuse, avec envahissement de la totalité du sinus maxillaire homolatéral. La lésion atteignait également la fosse nasale gauche ainsi que les cellules ethmoïdales antérieures gauches (Figs. 2a, 2 b et 3 ). L'artériographie a mis en évidence trois branches artérielles alimentant la tumeur: l'artère ophtalmique par les artères infra-orbitaires, l'artère maxillaire interne gauche et l'artère faciale gauche (Fig. 4). Après concertation, une prise en charge multidisciplinaire chirurgicale a été décidée. Une exérèse tumorale radicale avec
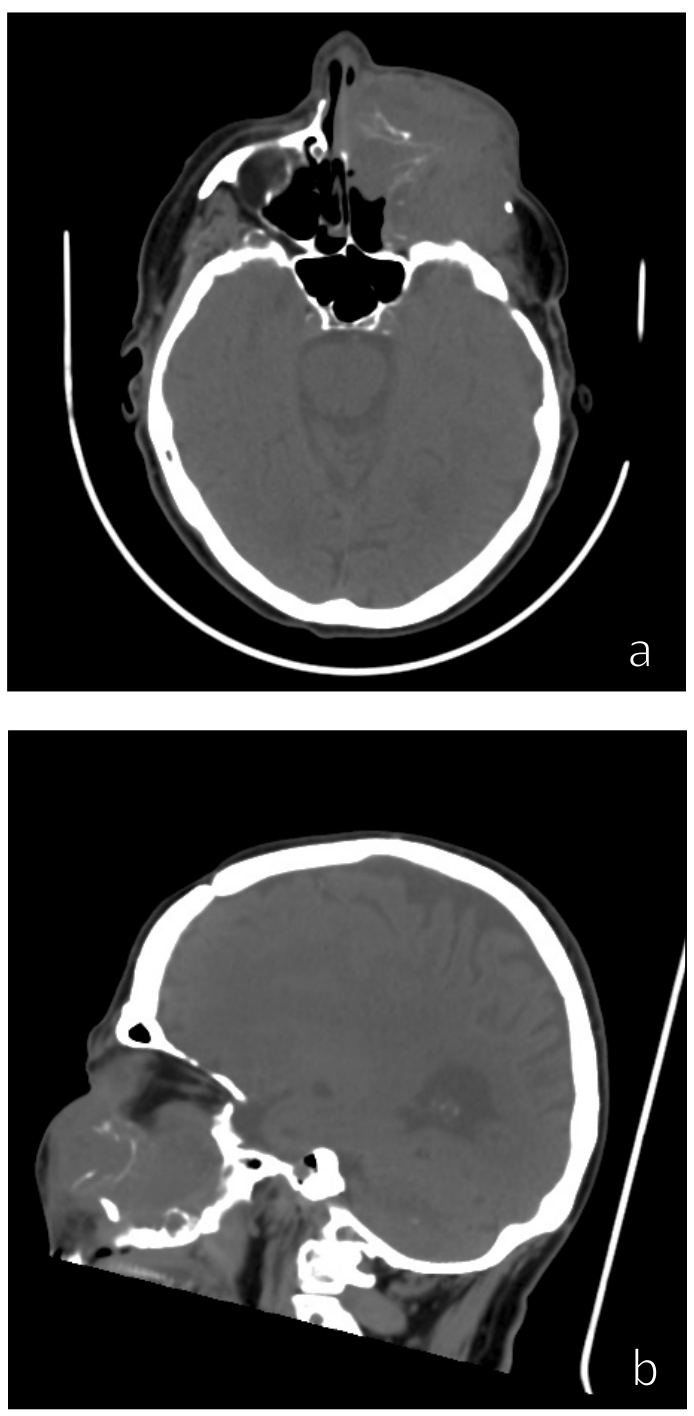

Fig. 2. CT-scan - coupe axiale (a) et coupe sagittale (b) - : tumeur ostéolytique, polylobée, intéressant le maxillaire gauche.

Fig. 2. CT-scan - axial view (a) and sagittal view (b) -: osteolytic polylobulated tumour involving the left side of the maxilla.

exentération de l'œil gauche a été réalisée. Préalablement la patiente a bénéficié, au sein du service de neuroradiologie, d'une artério-embolisation sélective pré-opératoire pour diminuer le risque de saignements durant l'intervention. Celle-ci a permis une dévascularisation de bonne qualité des apports provenant de la carotide externe (artère faciale, artère transverse de la face, artère maxillaire interne). L'embolisation des branches de l'artère ophtalmique a été réalisée à minima compte tenu de difficultés rencontrées, inhérentes à la technique. En fin d'embolisation, la dévascularisation était de bonne qualité pour le réseau carotidien interne (Fig. 5). Il persistait essentiellement un apport artériel provenant du siphon carotidien et des branches de l'artère ophtalmique, et à un plus 


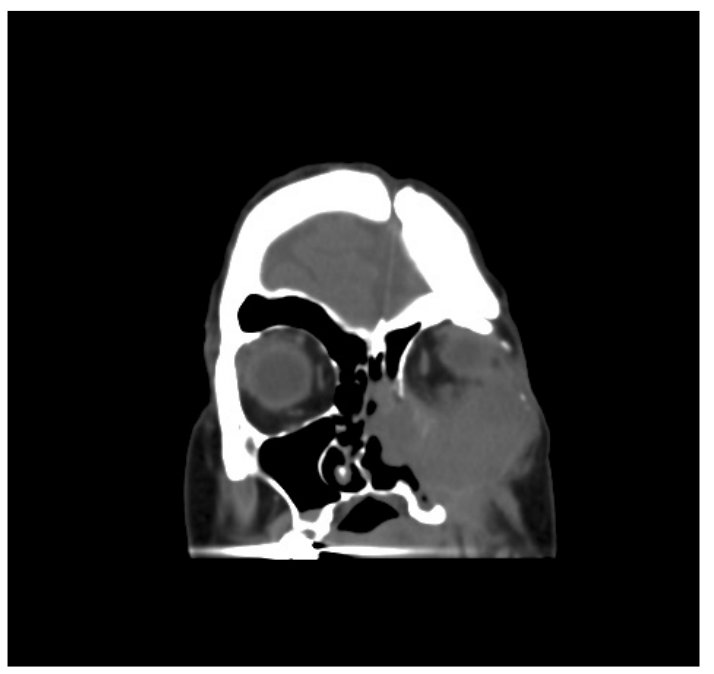

Fig. 3. CT-scan - coupe frontale - : envahissement de la fosse nasale gauche.

Fig. 3. CT-scan - coronal view -: invasion of the left nasal fossa.
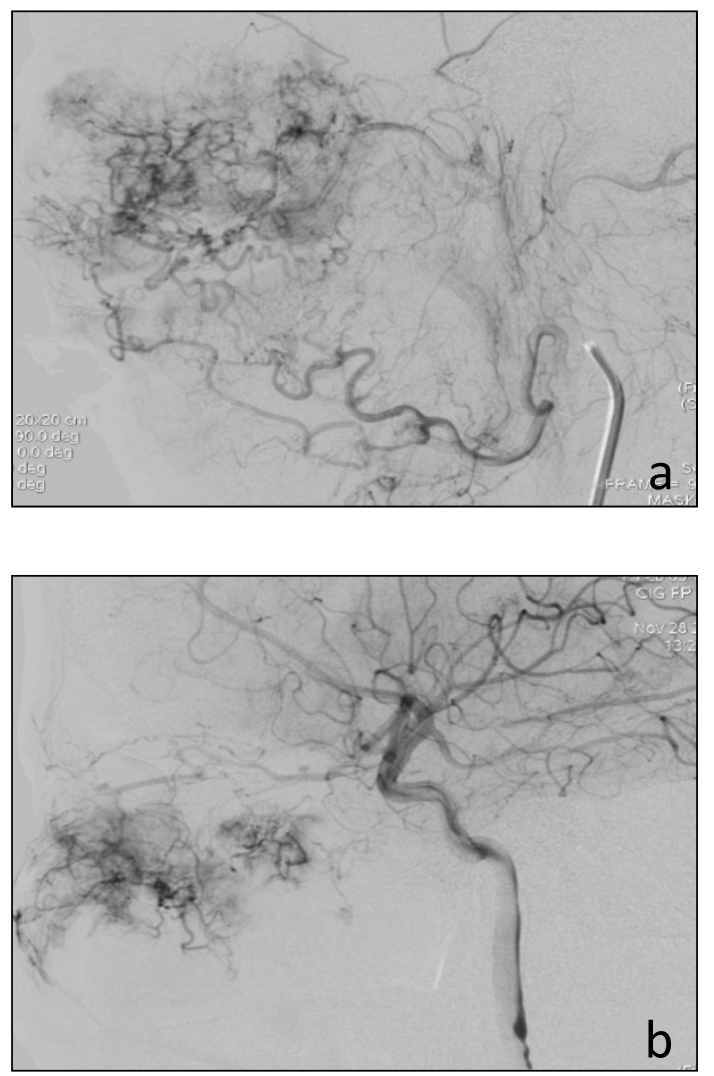

Fig. 4. Artériographie au temps précoce : visualisation du pédicule nourricier par l'artère carotide externe (4a) et par l'artère ophtalmique (4b) et des contours tumoraux « blush tumoral».

Fig. 4. Arteriography: an early arterial phase demonstrates the pedicle feeder arising from the left external carotid (4a) and from the ophthalmic artery (4b). The margins of the tumour are visualized "tumour blush".



Fig. 5. Artériographie après embolisation : dévascularisation du réseau carotidien externe et embolisation à minima des branches de l'artère ophtalmique.

Fig. 5. Arteriography after embolization: devascularisation of the external carotid network and a minima embolization of the ophthalmic artery branches.

faible degré de l'artère pharyngienne ascendante. La pièce de résection chirurgicale mesurait $7 \times 7 \times 5 \mathrm{~cm}$, avec le globe oculaire à son pôle supérieur. La tumeur blanchâtre mesurait $6,5 \times 4 \times 3,5 \mathrm{~cm}$ et présentait un aspect polylobé et des remaniements hémorragiques. Microscopiquement, la tumeur, partiellement lobulée par des cloisons collagènes hyalines, se caractérisait par une prolifération cellulaire assez dense, constituée de cellules monomorphes, avec de discrètes atypies et une densité modérée de mitoses ( 9 mitoses pour 10 champs au grossissement 40 ce qui correspond à $1.75 \mathrm{~mm}^{2}$ ). 0 n constatait de rares et petits foyers de nécrose tumorale. L'analyse immunohistochimique montrait une expression intense et diffuse par les cellules tumorales des antigènes CD34, bcl-2 et CD99, sans expression concomitante des kératines, de l'EMA, de la protéine $S 100$, des marqueurs musculaires (actine muscle lisse et desmine), ainsi que du CD31 et CD117. L'index prolifératif évalué à l'aide de l'anticorps Ki67 était mesuré à $10 \%$. Ce profil morphologique et immunohistochimique permettait de retenir le diagnostic de tumeur fibreuse solitaire du sinus maxillaire gauche avec une extension à la région orbitaire, présentant des critères histologiques d'agressivité.

\section{Discussion}

Les TFS surviennent chez des adultes d'âge moyen (sexe ratio $H: F=1$ ) [6]. Initialement décrites dans la plèvre sous le terme de mésothéliome bénin, la TFS a été rapporté dans les tissus mous dans de nombreuses localisations : l'orbite, les sinus de la face, le tractus respiratoire supérieur, le foie, la thyroïde, le sein, le tractus gastro-intestinal, la moelle épinière, les méninges et le médiastin. L'identité étant mieux connue, les TFS sont plus fréquemment diagnostiquées en situation extrapleurale que pleurale [7] ; les localisations pleurales ayant une 

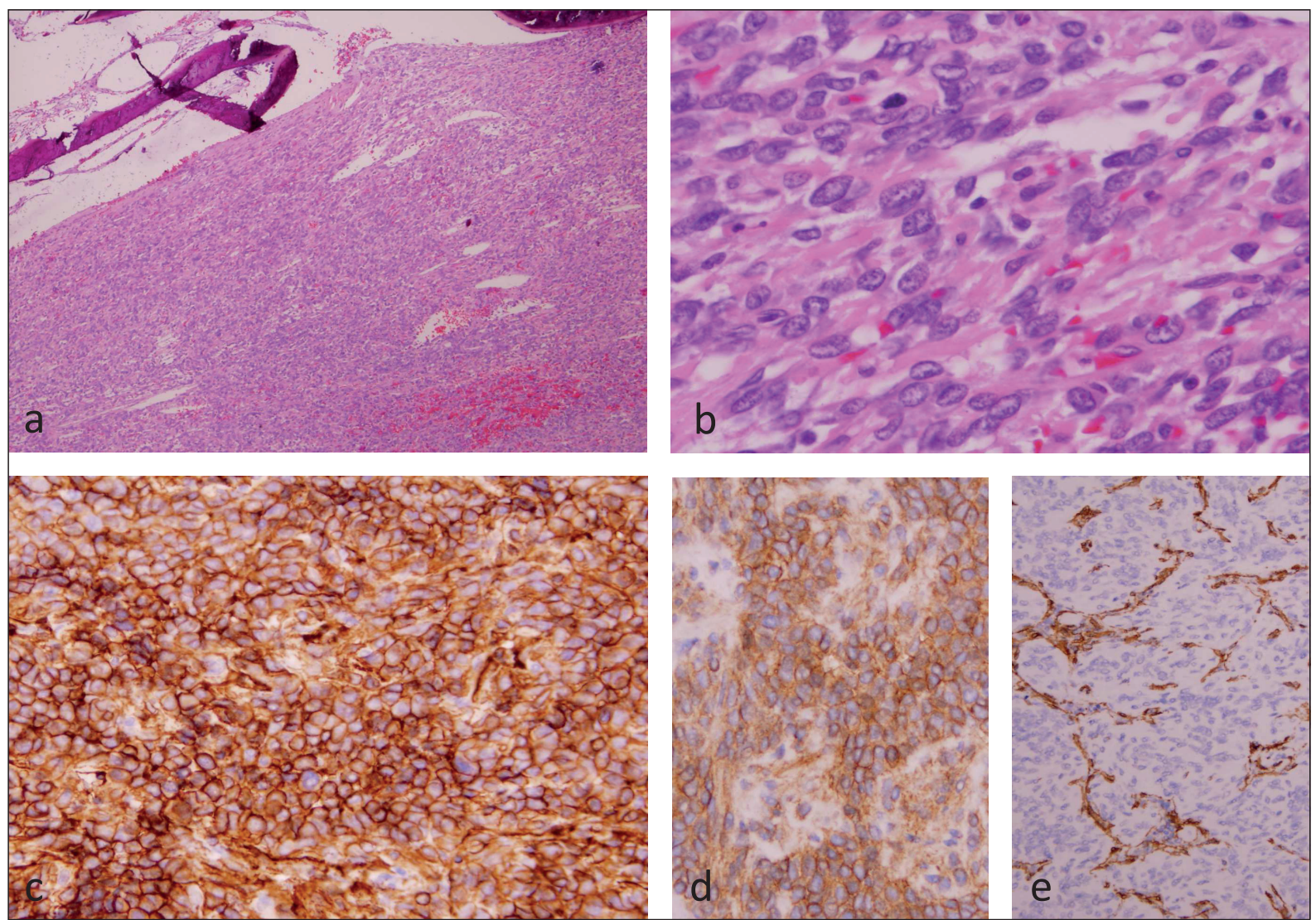

Fig. 6. Aspect histologique de la tumeur fibreuse solitaire : prolifération cellulaire assez dense de cellules monomorphes organisées en courts faisceaux ou sans agencement spécifique ( $a$ et b), expression intense et diffuse par les cellules tumorales des antigènes CD34 (c), CD99 (d) ; l'anticorps actine muscle lisse montre un riche réseau vasculaire qui n'a pas de caractère typiquement hémangiopéricytaire (e).

Fig. 6. Histological aspect of the solitary fibrous tumour: rather high cellular proliferation composed by monomorphic cells organized in short fascicles or without a specific pattern ( $a$ and $b$ ), tumoral cells show a strong and diffuse labelling for the CD34 and CD99 (d) antibodies; the antibody against the smooth muscle actin reveals an important vascular network without an hemangiopericytoma pattern (e).

évolution plus indolente que les formes extrapleurales. Les manifestations cliniques se limitent le plus souvent à une tuméfaction indolore, d'évolution lente. La peau ou la muqueuse peut présenter un érythème et/ou des télangiectasies. Un syndrome de masse est parfois observé, associant en fonction de la localisation tumorale des névralgies faciales du V2 ou du V3, un trismus, une baisse de l'acuité visuelle, des douleurs dentaires. Dans notre cas, les signes ophtalmologiques prédominaient du fait de la localisation et de son extension. Il existe quelquefois un syndrome paranéoplasique notamment une hypoglycémie [8] et plus rarement une ostéoarthropathie pneumique quand l'atteinte est pleurale ou pulmonaire. L'imagerie médicale (CT-scan et IRM) permet de confirmer la nature tumorale de la lésion, de préciser les limites et les rapports avec les structures du voisinage, ainsi que son caractère vasculaire par la prise de contraste. Le CT-scan retrouve une tumeur bien limitée, hétérogène, parfois lobulée, iso ou hyperdense. Des zones kystiques se mêlent aux zones nécrotiques. Un œdème péri-tumoral modéré peut-être retrouvé. Il existe un rehaussement intense après injection de produit de contraste iodé. La lyse osseuse est classique. L'IRM retrouve une image hétérogène, en iso signal $\mathrm{T} 1$ et plus rarement en hyper signal s'il existe une hémorragie intra-tumorale. Sur les séquences pondérées en T2, la lésion apparaît en iso signal ou hyper signal. Après injection de gadolinium, le rehaussement est précoce et intense. L'examen d'imagerie le plus révélateur est l'artériographie. Elle permet de réaliser une embolisation vasculaire. La tumeur admet en général deux artères nourricières dont le trajet est souvent modifié par la tumeur. Dans ce cas, il y avait trois branches : l'artère ophtalmique, l'artère maxillaire interne et l'artère faciale gauches. Avant de pénétrer dans la lésion, les artères forment un pédicule et se divisent en vaisseaux de 
calibres variables, organisés de façon radiaire telle une toile d'araignée [5]. Macroscopiquement, la lésion est richement vascularisée, relativement bien circonscrite, avec alternance de plages hémorragiques, nécrotiques et kystiques. Microscopiquement, la tumeur est composée de cellules à contours fusiformes, monomorphes, agencées en courts faisceaux ou sans pattern spécifique, avec un réseau de réticuline dense. L'analyse immunohistochimique constitue une aide précieuse pour le diagnostic différentiel. Les cellules tumorales expriment intensément dans la grande majorité des cas les antigénes CD34, bcl-2 et CD99, sans expression concomitante des cyto-

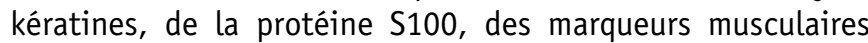
lisses, de CD117 et CD31 et des récepteurs hormonaux. Une hypercellularité, plus de 4 mitoses par 10 champs au grossissement $40\left(1.75 \mathrm{~mm}^{2}\right)$, des atypies nucléaires, ainsi que l'existence de zones de nécrose et d'hémorragies intra-tumorales seraient pour certains auteurs corrélées à un grade élevé de malignité [3]. La tumeur réunissait donc des critères histopathologiques d'agressivité : 9 mitoses par 10 champs, des petits foyers de nécrose et une cellularité diffusément élevée... La vascularisation souvent riche peut être de type dit hémangiopéricytaire ou plus anonyme. Bien que la majorité des TFS soit bénignes, le comportement clinique de la TFS peut être imprévisible. Plusieurs séries rapportent un taux de récidives locorégionales ou métastatiques allant de 9 à $19 \%$ dans les localisations extrapleurales, et pouvant survenir plus de 10 ans après la première lésion [4]. Pour certains auteurs, les récidives et les métastases seraient associées à certains caractères ou aspects histologiques. Ces particularités anatomiques sont : la taille $>10 \mathrm{~cm}$, l'hypercellularité, les atypies nucléo-cytoplasmiques marquées, la nécrose tumorale, les mitoses nombreuses (> 4 / 10 champs) et les marges infiltrantes. Il n'y a pas de corrélation stricte entre la morphologie et l'évolution clinique, toutefois la très grande majorité des TFS histologiquement bénignes ne récidivent pas et la grande majorité des tumeurs histologiquement agressives vont également avoir un comportement clinique agressif. De nombreuses études rappelent que le principal critère prédictif d'une évolution favorable est l'exérèse complète de la lésion.

La TFS peut être difficile à diagnostiquer du fait de sa similitude avec d'autres tumeurs des tissus mous. Le diagnostic différentiel se fait avec les tumeurs fuso-cellulaires et les pseudotumeurs notamment l'hémangiopéricytome (HPC). Il faut noter que la vascularisation de type hémangiopéricytaire n'est pas spécifique de la TFS et peut se voir dans plusieurs autres tumeurs. Le diagnostic différentiel avec l'hémangiopéricytome est parfois difficile car les deux tumeurs peuvent avoir le même profil immunohistochimique. Toutefois, l'HPC présente une vascularisation plus riche et tend à présenter un marquage moins intense avec le CD34. Récemment, l'hémangiopéricytome a été reclassé comme une variante de la TFS $[9,10]$. Les signes cliniques de TFS sont non spécifiques. L'imagerie (CT-scan et IRM) permet de préciser l'extension de la lésion et ses limites. Seul l'examen anatomopathologique peut trancher et donner le diagnostic exact grâce à l'histologie et à l'immunohistochimie. L'artériographie est un examen de choix dans la prise en charge pour le diagnostic et le traitement. Compte tenu de leur évolution imprévisible, les TFS nécessitent une surveillance à long terme.

Conflits d'intérêt : aucun

\section{Références}

1. Stunt AP, Muray M. Hemangiopericytoma, a vascular tumor featuring Zimmerman's pericyte. Ann Surg 1942;116:26-33.

2. Dufour H, Bouillot P, Figarella-Branger D, Nodoye N, Regis J, Njee Bugha T, Grisoli F. Hémangiopéricytomes méningés. Revue rétrospective de 20 cas. Neurochirurgie 1998;44:5-18.

3. Mena H, Ribas JL, Pezeshkpour GH, Cowan DN, Parisi JE. Hemangiopericytoma of the central nervous system: a review of 94 cases. Hum Pathol 1991;22:84-91.

4. Winek RR, Rcheithauer BW, Wick MR. Meningioma, meningeal hemangiopericytoma (angioblastic meningioma), peripheral hemangiopericytoma, and acoustic schwannoma. A comparative immunohistochemical study. Am J Surg Pathol 1989;13:251-61.

5. Greenfield GB. Radiology of bone diseases (pp. 532-3). JB Lippincott, Philadelphia 1990.

6. Desser TS, Stark P. Pictorial essay. Solitary fibrous tumors of the pleura. J Thorax Imaging 1998;13:27-35.

7. Duffaut-Andreux C, De Pinieux G, Guerini H, Malan S, Pessis E, Godefroy D, Drapé J-L, Toméno B, Chevrot A. Tumeurs fibreuses solitaires des parties molles : à propos de 4 cas. J Radiol 2004;85:1537.

8. Bouchi J, Gharios E, Cortbawi E, Aftimos G. Tumeur fibreuse solitaire de la plèvre avec coma et hypoglycémie. Rev Pneumol Clin 1993;49:243-6.

9. Graadt van Roggena JF, Hogendoorna PCW. Solitary fibrous tumour: the emerging clinicopathologic spectrum of an entity and its differential diagnosis. Current Diagnostic Pathology 2004;10:229-35.

10. Wat SY, Sur M, Dhamanaskar K. Solitary fibrous tumor (SFT) of the pelvis. Clin Imaging 2008;32:152-6. 